\title{
Pattern formation in diffusive-advective coupled map lattices
}

\author{
Pedro G. Lind, ${ }^{1,2,3,4, *}$ João Corte-Real, ${ }^{2,3}$ and Jason A. C. Gallas ${ }^{1,2,3,4, \dagger}$ \\ ${ }^{1}$ Instituto de Física, Universidade Federal do Rio Grande do Sul, 91501-970 Porto Alegre, Brazil \\ ${ }^{2}$ Unidade de Meteorologia e Climatologia, Instituto de Ciência Aplicada e Tecnologia, Faculdade de Ciências, \\ Universidade de Lisboa, 1749-016 Lisboa, Portugal \\ ${ }^{3}$ Centro de Geofísica, Universidade de Évora, 7000 Évora, Portugal \\ ${ }^{4}$ Institut für Computer Anwendungen, Universität Stuttgart, Pfaffenwaldring 27, D-70569 Stuttgart, Germany
}

(Received 8 October 2003; published 4 June 2004)

\begin{abstract}
We investigate pattern formation and evolution in coupled map lattices when advection is incorporated, in addition to the usual diffusive term. All patterns may be suitably grouped into five classes: three periodic, supporting static patterns and traveling waves, and two nonperiodic. Relative frequencies are determined as a function of all model parameters: diffusion, advection, local nonlinearity, and lattice size. Advection plays an important role in coupled map lattices, being capable of considerably altering pattern evolution. For instance, advection may induce synchronization, making chaotic patterns evolve periodically. As a byproduct we describe a practical algorithm for classifying generic pattern evolutions and for measuring velocities of traveling waves.
\end{abstract}

DOI: 10.1103/PhysRevE.69.066206

PACS number(s): 89.75.Kd, 05.45.Xt, 05.45.Ra

\section{INTRODUCTION}

The study of the intricacies underlying pattern formation and pattern dynamics allows one to probe the nonlinear mechanisms of nonequilibrium conditions in many physical phenomena such as, laser dynamics [1], synchronization of optical patterns [2], electroconvection [3], rheological phenomena in fluid systems [4], field-induced phenomena in magnetic fluids [5], oscillatory and translational Turing patterns in reaction-diffusion systems [6-8], Rayleigh-Bénard convection in hydrodynamical systems [9-11], and many others [12]. Pattern formation is of importance also for biological systems, e.g., in investigations of pattern formation inside living cells [13] and morphological structures and self-organized patterns in bacteria colonies [14,15].

A very popular way of modeling pattern formation is by using lattices of coupled oscillators ruled locally either by differential equations or by time-discrete mappings, the socalled coupled map lattices [16,17], models which are realistic and consume considerably less computer time. For instance, coupled map lattices with chaotic elements coupled through their amplitudes and phase have been recently proposed as a model of a gas [18]. They also provide suitable models of information coding in nervous systems [19]. Other useful applications include the study of wavelike solutions in diffusive lattices $[20,21]$, of pattern selection induced by external forcing [22], reaction-diffusion processes in hierarchical structure [23], ocean convection parametrization [24], synchronization processes of patterns [25-27], and many others [16].

Traditionally, patterns are studied considering only the competition between local nonlinearities together with the amplitude spreading due to diffusion. However, as is well

\footnotetext{
*URL: http://www.ica1.uni-stuttgart.de/ lind

${ }^{\dagger}$ URL: http://www.ica1.uni-stuttgart.de/ jgallas
}

known [28], spatially extended phenomena are quite frequently subject not only to diffusion but also to advection, particularly in ocean circulation and climate [29]. Denoting by $\gamma$ the advection strength, a simple model incorporating advection was proposed recently [30,31]:

$$
x_{t+1}(i)=f\left(x_{t}(i)\right)+\varepsilon \mathcal{D}_{i, t}-\gamma \mathcal{A}_{i, t},
$$

where, as usual, $\varepsilon$ represents the diffusion, $f(x)$ controls the local dynamics, $\mathcal{D}_{i, t}$ and $\mathcal{A}_{i, t}$ are discretized forms of the diffusion and advection operators, respectively,

$$
\begin{gathered}
\mathcal{D}_{i, t}=\frac{f\left[x_{t}(i+1)\right]+f\left[x_{t}(i-1)\right]}{2}-f\left[x_{t}(i)\right], \\
\mathcal{A}_{i, t}=\frac{f\left[x_{t}(i+1)\right]-f\left[x_{t}(i-1)\right]}{2}
\end{gathered}
$$

Models similar to Eq. (1) above have been used before to investigate anisotropic coupling schemes in lattices of maps and open flows [32-35]. In our model, however, the asymmetry parameter $\gamma$ corresponds to an advective velocity $[30,31]$. In the following we consider periodic boundary conditions only.

The purpose of this paper is to report a detailed investigation of the role of advection in pattern formation based on the diffusive-advective model of Eq. (1). First, we describe a general algorithm for identifying the propagation of periodic patterns in lattices of maps and measuring their period and velocity. The distribution of patterns is then studied as a function of diffusion and advection as well as nonlinearity and lattice size. We show that advection plays an important role and is capable of considerably changing pattern behaviors. In particular, in certain parameter ranges, advection may change chaotic into regular (periodic) behavior. 
The paper is organized as follows. In Sec. II we digress about technical matters necessary to characterize "identical patterns" for discrete-time evolutions in lattices and use it to classify pattern evolutions into five generic classes, three periodic and two nonperiodic. In Sec. III we report the relative distribution (histograms) of patterns as a function of the local nonlinearity of the logistic map, the diffusion $\varepsilon$, the advection $\gamma$, and the lattice size $L$. Discussion and conclusions are given in Sec. IV.

\section{RECOGNIZING IDENTICAL PATTERNS}

The key concept when studying spatially extended systems is that of "pattern." Roughly speaking, a pattern is a snapshot showing the configuration of the system at a given instant. For spatially continuous systems, a pattern is a continuous function [12], say $\xi(r)$ with $r \in \mathbb{R}$. For spatially discrete systems (lattices), a pattern is a discrete set of amplitudes $x_{t}(i)$, which we represent by $\mathcal{P}_{t} \equiv\left\{x_{t}(i)\right\}$, where $i \in \mathbb{N}$. Note that while the amplitudes $\xi(r)$ always vary continuously at each point $r$ for lattices, the amplitudes $x_{t}(i)$ at each site $i$ may be either a discrete set of values, like in cellular automata, or a continuous interval, like in coupled map lattices. We call indistinctly space of states the set of all possible local amplitudes that a system may assume.

After introducing these notions, the next important thing is to be able to identify whether two given patterns are identical or not. The identification would be trivial if patterns could never appear spatially shifted on the system. For continuous systems the detection of identical patterns is rather simple: two continuous patterns $\xi(r)$ and $\eta(r)$, are identical when it is possible to find a constant $\delta \in \mathbb{R}$ such that $\eta(r)$ $=\xi(r-\delta)$ for all $r$. Obviously, when $\delta=0$ the patterns coincide, i.e., they are not shifted with respect to each other.

For discrete systems two cases need to be distinguished since the space of states may be either discrete (cellular automata) or continuous (lattices of maps). For cellular automata, the definition is similar to that for continuous systems: patterns are identical if $x_{t}(i)=x_{t}(i-\delta)$, for all $i$ where now $\delta$ is an integer, not a real number, since space and space of states are both discrete.

For lattices of maps, the situation is slightly more subtle since direct comparison of the actual amplitudes may not necessarily reveal their identity. As illustrated in Fig. 1, to recognize if two patterns $\left\{X_{1}, X_{2}, X_{3}, X_{4}\right\}$ and $\left\{Y_{1}, Y_{2}, Y_{3}, Y_{4}\right\}$ are identical requires comparing auxiliary interpolated continuous functions, say $\mathcal{I}(r)$ and $\mathcal{I}_{1}(r)$. Although patterns appear to be completely different because their amplitudes are different, the interpolated functions $\mathcal{I}(r)$ and $\mathcal{I}_{1}(r)$ associated with them are identical in the sense that $\mathcal{I}_{1}(r)=\mathcal{I}(r-\delta)$ for all $r$. Therefore, in sharp contrast with the situation for continuous systems and for cellular automata as discussed above for lattices we need to compare the auxiliary continuous functions. The auxiliary continuous functions are needed because although space is discrete, the space of states (amplitudes) is continuous.

To define the auxiliary continuous function we use a convenient basis set $\left\{f_{k}(r)\right\}$. Two patterns $\left\{X_{i}\right\}$ and $\left\{Y_{i}\right\}$ are iden-

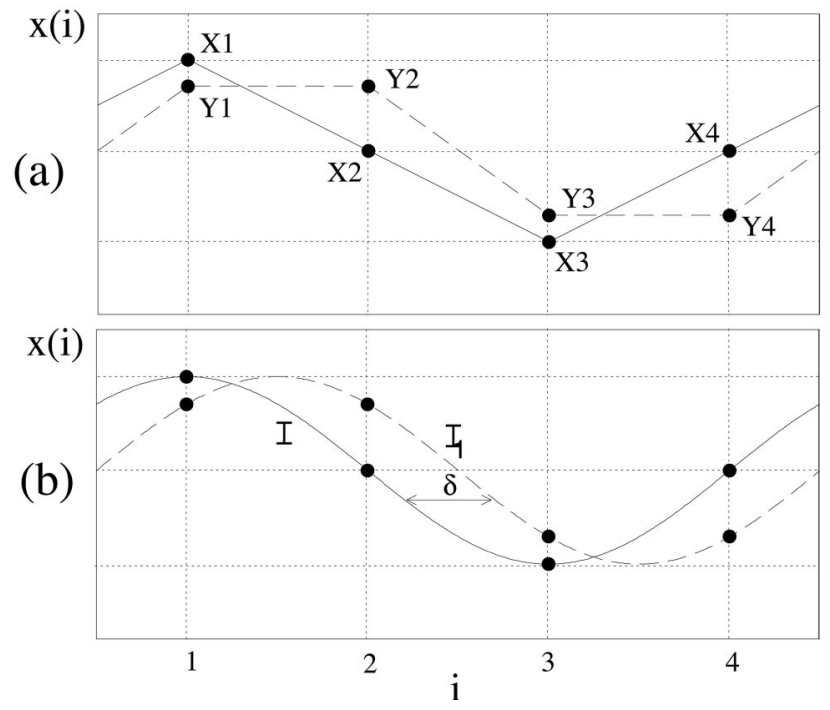

FIG. 1. The definition of identical patterns in discrete lattices cannot be always made with the actual amplitudes but may require finding an interpolated function. (a) No translation of the amplitudes $\left\{X_{1}, X_{2}, X_{3}, X_{4}\right\}$ make them coincide with the amplitudes $\left\{Y_{1}, Y_{2}, Y_{3}, Y_{4}\right\}$. Straight lines are simple guides to the eye here; (b) the interpolated functions, $\mathcal{I}(r)$ and $\mathcal{I}_{1}(r)$ show that amplitudes are in fact correlated. See text.

tical if and only if their corresponding interpolated functions $\mathcal{I}(r)=\sum_{k=0}^{L-1} A_{k} f_{k}(r)$ and $\mathcal{I}_{1}(r)=\sum_{k=0}^{L-1} B_{k} f_{k}(r)$, satisfy for all $r$ the condition

$$
\mathcal{I}(r)=\mathcal{I}_{1}(r-\delta),
$$

for some fixed $\delta \in \mathbb{R}$.

Obviously, the above definition of identical patterns applies also for spatially continuous systems, when only a single function is needed (the pattern itself), as well as for cellular automata. Next, we apply this definition to detect temporal repetitions of patterns, shifted or not.

As time evolves in discrete steps, the lattice displays a sequence of patterns

$$
\mathcal{P}_{t_{0}}, \mathcal{P}_{t_{1}}, \ldots, \mathcal{P}_{t_{\ell}}, \ldots
$$

each one having an interpolating function associated with it:

$$
\mathcal{I}_{t_{0}}, \mathcal{I}_{t_{1}}, \ldots, \mathcal{I}_{t_{\ell}}, \ldots
$$

If the initial pattern $\mathcal{P}_{t_{0}}$ reappears at some later time $t_{\ell}$, their interpolated functions, $\mathcal{I}_{t_{0}}$ and $\mathcal{I}_{t_{\ell}}$ must obey the condition of Eq. (3). In the particular case when $\delta=0$ the amplitudes of both patterns coincide identically. In all other cases, when $\delta \neq 0$, despite having different amplitudes, the patterns $\mathcal{P}_{t_{0}}$ and $\mathcal{P}_{t_{\ell}}$ are identical since, according to Eq. (3), they are embedded in the same interpolating function. In any case the velocity $v$ of the pattern is $v=\delta /\left(t_{\ell}-t_{0}\right)$.

To implement the above definition of identical patterns for a lattice composed by $L$ sites one needs to solve a linear system of $L$ equations at each time step and compare the corresponding solutions (coefficients). However, a more con- 

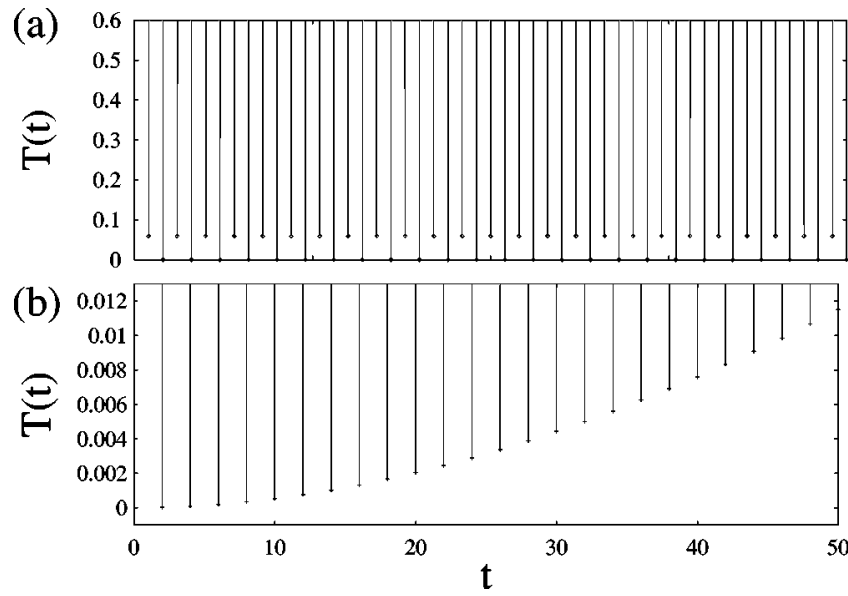

FIG. 2. Illustrative examples of $T(t)$, Eq. (6), characterizing the possible nature of the periodic behaviors: (a) static patterns $(\delta=0)$, when $T\left(t_{\ell}\right) \equiv 0$, (b) moving patterns $(\delta \neq 0)$, when $T\left(t_{\ell}\right) \simeq 0$. The values $T\left(t_{\ell}\right)$ increase quadratically with $t$.

venient and fast procedure is to use an equivalent numerical algorithm based on least squares deviations of the amplitudes both in time and in space.

To this end we fix a reference pattern, say $\mathcal{P}_{t_{0}}=\left\{x_{t_{0}}(i)\right\}$, and compute the quantity

$$
T(t)=\sum_{i=1}^{L}\left[x_{t}(i)-x_{t_{0}}(i)\right]^{2}
$$

This function is useful because $T\left(t_{\ell}\right) \simeq 0$ for all instants $t_{\ell}$ for which the pattern of reference reappears, thereby allowing one to determine the period $\tau$. Note that one finds $T\left(t_{\ell}\right) \equiv 0$ only for static patterns, i.e., when $\delta=0$. To have a moving pattern implies having $\delta \neq 0$ and, therefore, to have $T\left(t_{\ell}\right)$ $\simeq 0$. The difference between static and moving patterns is illustrated in Fig. 2.
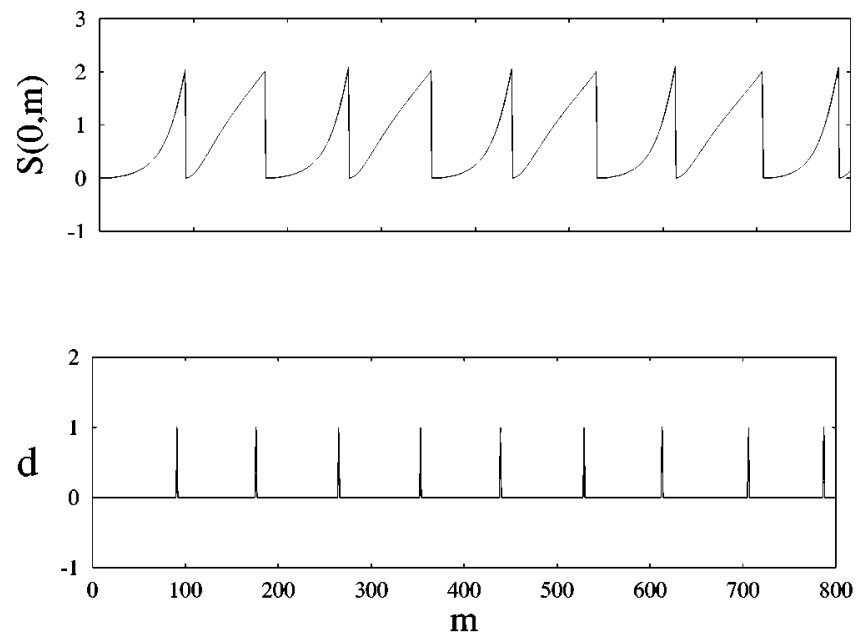

After determining the period $\tau$ instead of working in the "fast scale" $t$, we may simply consider patterns at instants which are multiples of $\tau$, namely $m \tau$, using the "slow scale" $m$. With the slow scale $m$ we determine the direction of movement through the function

$$
S(j, m)=\sum_{i=1}^{L}\left[x_{t_{0}+m \tau}(j+i)-x_{t_{0}}(i)\right]^{2},
$$

where $j$ and $m \in \mathbb{N}$, and the sum $i+j$ is taken modulo $L$. Obviously, for $j=0$ we have $S(0, m) \equiv T(m \tau)$.

Equation (7) allows us to define a useful "displacement indicator" $d_{m}$ as being the first $j$ which minimizes $S(j, m)$. Obviously, $d_{m}$ must be an integer between 0 and $L$, the size of the lattice. To detect a moving pattern means to detect a nonzero value of $d_{m}$. Since patterns generally move quite slowly, one typically detects $d_{m}=0$ during a relatively long interval before finding $d_{m} \neq 0$. Since whenever $d_{m}$ is nonzero our algorithm updates the reference and computes $d_{m+1}$, for any $m$ only three values may be found: $d_{m}=0,1, L-1$. A pattern is said to be moving in the "positive" direction whenever $d_{m}=1$, and in the "negative" when $d_{m}=L-1$.

Figure 3 illustrates typical $S(j, m)$ and $d$ for moving patterns [Fig. 3(a)] and chaotic pattern evolutions [Fig. 3(b)]. For static patterns (not shown), $d$ is always zero, i.e., the minimum of $S(j, m)$ is always reached for $j=0$.

With the period $\tau$ and displacement indicator $d_{m}$ we are able to detect and classify any pattern evolution (PE). The classification is as follows.

If a PE has period $\tau$ periodic, then the associated $\tau$ patterns either remain static ( $d_{m}=0$ always), or not. Thus, as shown in Fig. 4, there are three classes of periodic PEs: static (class $S$ ), positively moving (class $P$ ), and negatively moving patterns (class $N$ ).

We find convenient to separate nonperiodic PEs in two broad classes: truly chaotic evolutions (class $C$ ) or chaotic evolutions consisting of PE during finite intervals of time.
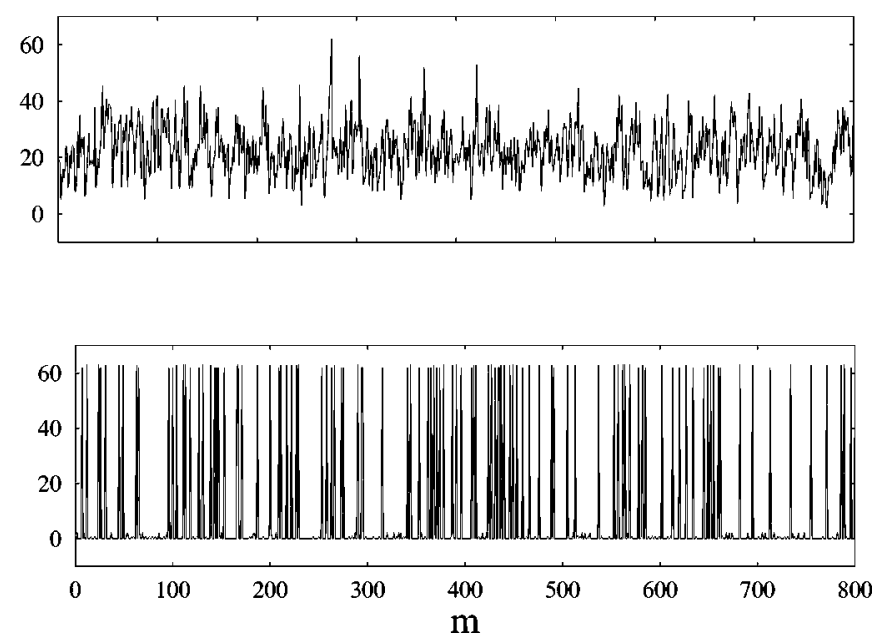

FIG. 3. Typical temporal variation of $S(j, m)$ for $j=0$, and of $d$. Left column: positive moving pattern $[a=1.73, \varepsilon=0.5, L=64]$. Right column: chaotic pattern evolution $[a=1.95, \varepsilon=0.5, \gamma=0, L=64]$. For negative moving patterns (not shown) one obtains results, with nonnegative values of $d$ being $d_{m}=L-1$. 

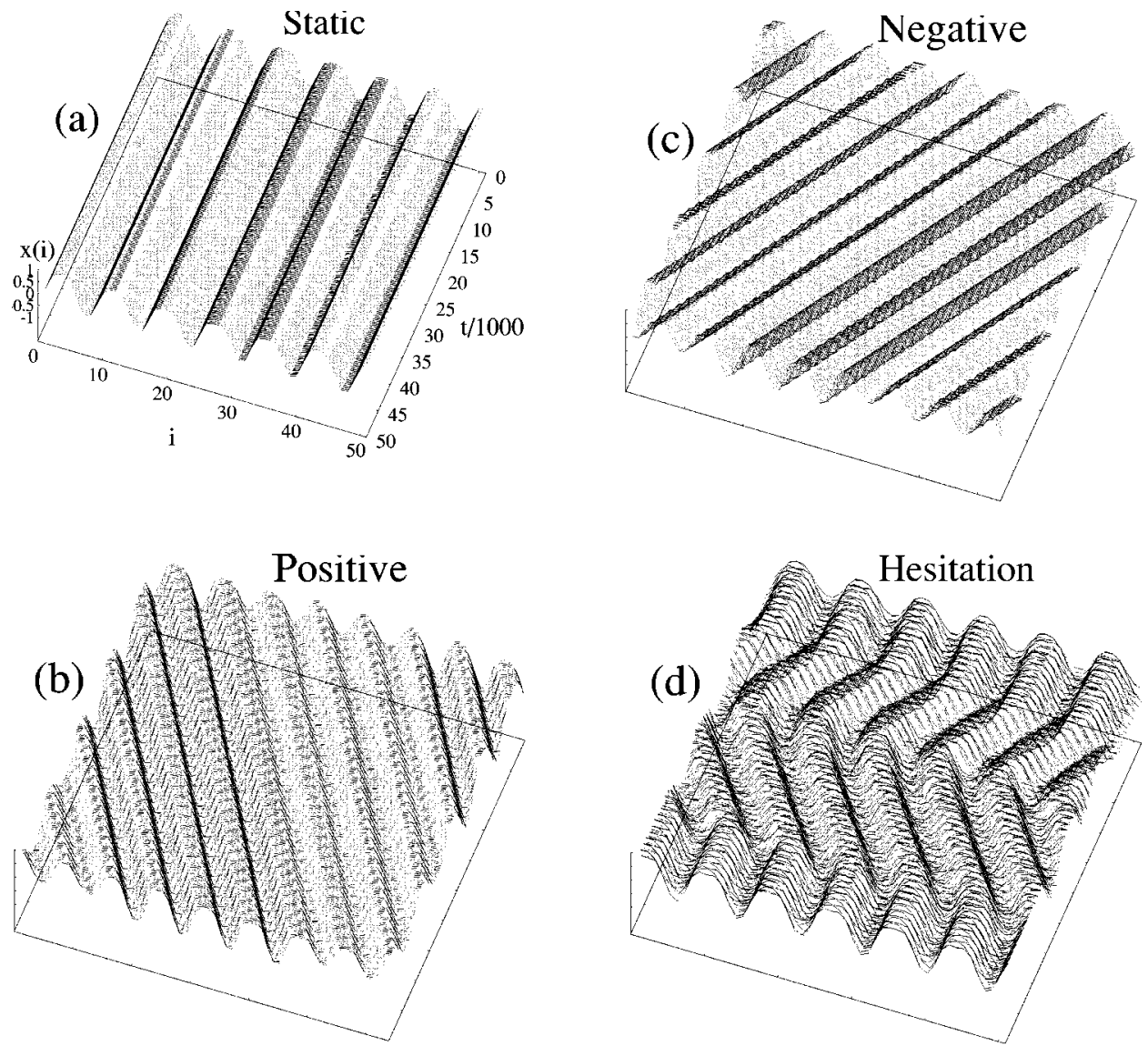

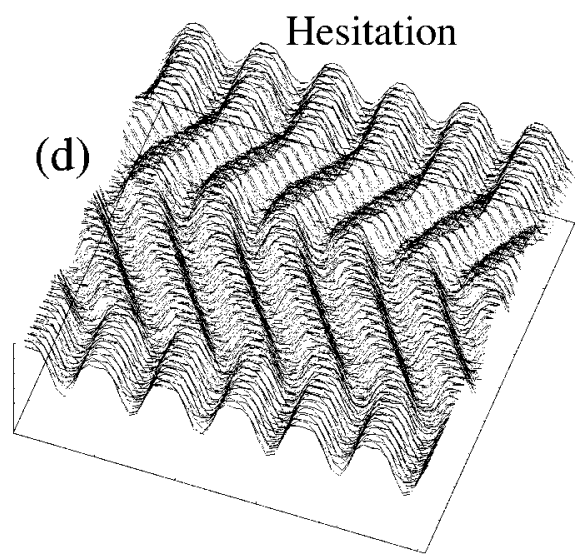

FIG. 4. Illustrative examples of the five classes of pattern evolutions for the diffusive-advective coupled map lattice: (a) Class $S$, "static," (b) Class $P$, "positively" moving; (c) Class $N$, "negatively" moving; (d) Class $H$, "hesitation"; and (e) Class C, "chaotic". For clarity, adjacent points of each pattern are joined with lines.

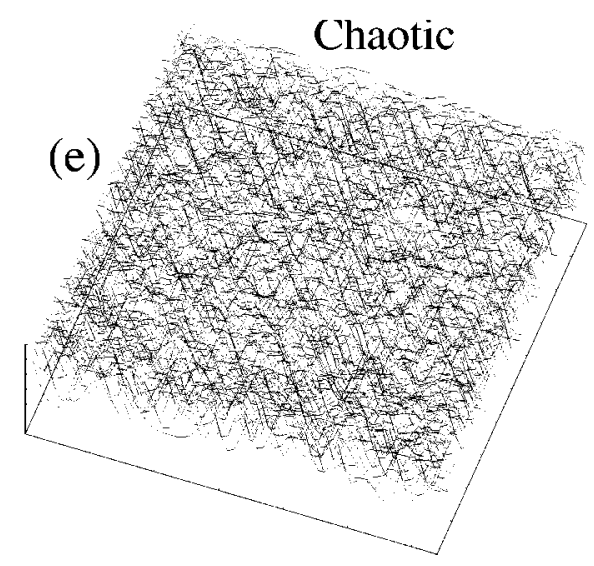

We refer to this situation as Hesitating pattern evolutions (class $H$ ), see Fig. 4(d).

When implementing our algorithm, it is useful to consider the following additional points. First, to avoid spurious local minima, the computation of $\tau$ requires a majorant $\mathcal{M}$ of $T(t)$. This majorant depends on the local dynamics $f(x)$. An adequate choice for the logistic map is $\mathcal{M}=0.2$. Second, to prevent neglecting a local minima of a moving pattern, we impose that $T\left(t_{0}+m \tau\right)$ and $T\left[t_{0}+(m+1) \tau\right]$ are of the same order of magnitude. Third, whenever a value for $\tau$ is found, the reference $\left\{x_{t_{0}}(i)\right\}$ is updated, and a new value of $\tau$ is determined and compared with the preceding one. This procedure is repeated during an interval of $\mathcal{T}$ time steps. The accuracy of $\tau$ increases when $\mathcal{T}$ increases, up to a point when
$\mathcal{T}$ is so large that PEs with high velocities, of the order of $v \sim 1$ site/step, may complete a full revolution around the lattice in less than $\mathcal{T}$ time steps. In our cases, $\mathcal{T} \sim 10^{3}$ time steps proved to be an adequate choice.

Finally, note that while for continuous time evolutions it is always possible to determine the direction of motion by successive measurements of the position at $t_{n}=t_{n-1}+\left(t_{n-1}\right.$ $\left.-t_{n-2}\right) / 2$, for discrete times one has the restriction $t_{n}-t_{n-1}$ $\geqslant 1$. Therefore, during one time step, the displacement of $p$ sites in one direction or, equivalently, of $L-p$ sites in the opposite direction, are both possible. To determine the actual direction of motion, one must take into account that, since we have nearest neighbors coupling, state propagation cannot be greater than one site per time step. Consequently, after 
TABLE I. Characterization of the five classes using $\tau$ and $d_{m}$. Class $H$ consists of an alternation of periodic and chaotic behaviors which occur during finite time intervals.

\begin{tabular}{lccccc}
\hline \hline & Class & Period $\tau$ & Displacement $d_{m}$ & $S_{m}=L-\left|L-2 d_{m}\right| / 2$ & Direction $\sigma_{m}$ \\
\hline Peiodic & Static & Constant & 0 & 0 & \\
& Positive & Constant & 1 & 1 & +1 \\
& Negative & Constant & $L-1$ & 1 & -1 \\
\multirow{4}{*}{ Nonperiodic } & Hesitation & Constant/undefined & $(0,1, L-1) /$ undefined & $(0,1) /$ undefined & $\pm 1 /$ undefined \\
& Chaos & undefined & undefined & undefined & undefined \\
\hline \hline
\end{tabular}

one period ( $\tau$ time steps) the pattern cannot move more than $\tau$ sites. Thus, when $0 \leqslant p \leqslant \tau$ we say that the pattern is moving in a positive direction, while when $L-\tau \leqslant p \leqslant L-1$ it is said to be moving in the opposite negative direction.

The velocity $v$ of moving patterns may be directly computed from $\tau$ and $d_{m}$ by averaging over a time interval $M \tau$ the quantity $\left(L-\left|L-2 d_{m}\right|\right) / 2$, weighted by the sign $\sigma_{m}$ of $L$ $-2 d_{m}$ which indicates the direction of movement:

$$
v=\frac{1}{2 M \tau} \sum_{m=1}^{M} \sigma_{m}\left(L-\left|L-2 d_{m}\right|\right) .
$$

After some elementary algebra one sees that $(L-\mid L$ $\left.-2 d_{m} \mid\right) / 2$ equals the displacement for each $d_{m}$, independently of the direction in which the pattern moves. In particular, this quantity has the same value for both directions, positive $\left(d_{m}=1\right)$, and negative $\left(d_{m}=L-1\right)$, and is zero for $d_{m}=0$. Note that, if $\sigma_{m}$ has always the same value, say $\sigma$, then the PE belongs to class $P(\sigma=1)$ or to class $N(\sigma=-1)$, while if $\sigma$ $=1$ and -1 intermittently, the PE belongs to class $H$ and the velocity (8) has the meaning of an average velocity.

A summary of the classification is given in Table I. Looking at this classification, one may wonder if Table I should not also include quasiperiodic solutions. The problem here is how to unambiguously characterize the presence of quasiperiodicity in coupled map lattices. The term "quasiperiodicity" was already used by Kaneko [36] and Franceschini and Vernia [37], but without a proper definition of what should be precisely understood by it. Subsequently, Franceschini et al. discussed solutions which they call "quasiperiodic traveling waves," characterizing them by a winding number $\omega=1 / J$, where $J$ is the amount of time steps needed for a site to complete one turn in phase-space. We feel that the term quasiperiodic traveling waves is somewhat contradictory and prone to confusion. In a lattice, we understand that one either has a "traveling wave," when a wavelike pattern repeats periodically in time but shifted in the lattice, or "quasiperiodic solutions," when it is possible to associate an irrational winding number to the solution. Since the characterization of irrational winding numbers for spatially extended system is not at all trivial, not to say impossible, we find more appropriate to talk generically about traveling waves only, while we wait for a reliable way (algorithmic, not conceptual) of recognizing quasiperiodicity in spatially extended systems.

\section{CHARACTERIZATION OF PATTERN EVOLUTIONS}

In this section we apply the algorithm above to characterize and classify PEs in the diffusive-advective model, Eq. (1), for the usual quadratic local dynamics

$$
x_{t+1}=f\left(x_{t}\right)=1-a x_{t}^{2} .
$$

In Sec. III A we consider the purely diffusive regime $(\gamma=0)$ while in Sec. III B we consider the effect of advection. We compute histograms for all possible combinations of parameters using samples of 100 random initial conditions. For fixed lattice size $L$ we use a mesh of $50 \times 50$ grid points, and a mesh of $100 \times 50$ grid points when varying $L$ among 100 different values. In both cases the grid resolution is sufficient to catch the main features of the distribution of the PEs. Pattern evolutions are classified after discarding transients of $10^{5}$ time steps, being carried out during $10^{4}$ subsequent time steps. A summary of all the results found is given in Table II, at the end of this section. We now discuss these results in detail.

\section{A. The purely diffusive lattices}

The results of this section extend preliminary work [39] and serve as reference to compare the modifications caused by including advection.

Figure 5 shows the distribution of pattern evolutions as a function of $a$ and $\varepsilon$ for the same lattice size $L=64$ considered earlier by Kaneko and Tsuda [16]. For $a \lesssim 1.4$ we find a "plateau" where only periodic PEs are observed. The upper boundary of this plateau lies approximately at the accumulation point $a_{\infty} \simeq 1.4011$ of the $2^{\ell}$ doubling cascade of the local map, Eq. (9). This "periodic plateau," extends until $a$ $=-0.25$, the lower limit of allowed $a$ values. The periodic plateau is composed only by static (class $S$ ) PEs, except for $1.27 \lesssim a<a_{\infty}$ and $\varepsilon \gtrsim 0.45$, where pattern evolutions belonging to class $P$ and $N$ are also observed. The lower limit $a$ $\sim 1.27$ corresponds approximately to $2 \rightarrow 4$ doubling bifurcation of the local quadratic map. Although period-4 plays a crucial role in traveling wave (TW) solutions for coupled map lattices [40], as far as we know there is no complete explanation for the absence of moving patterns below the period-4 bifurcation. Moving patterns observed in all these regions have low velocities, $v \sim 10^{-4}$ site/step.

Beyond the periodic plateau (for $a>a_{\infty}$ ) nonperiodic classes predominate, except in the region $1.6 \lesssim a \lesssim 1.9$ when $\varepsilon \gtrsim 0.45$ and $a \gtrsim 1.6$ for $\varepsilon \sim 0.15$, where traveling waves are profusely observed, with velocities $v \sim 10^{-3}$ site/step. 

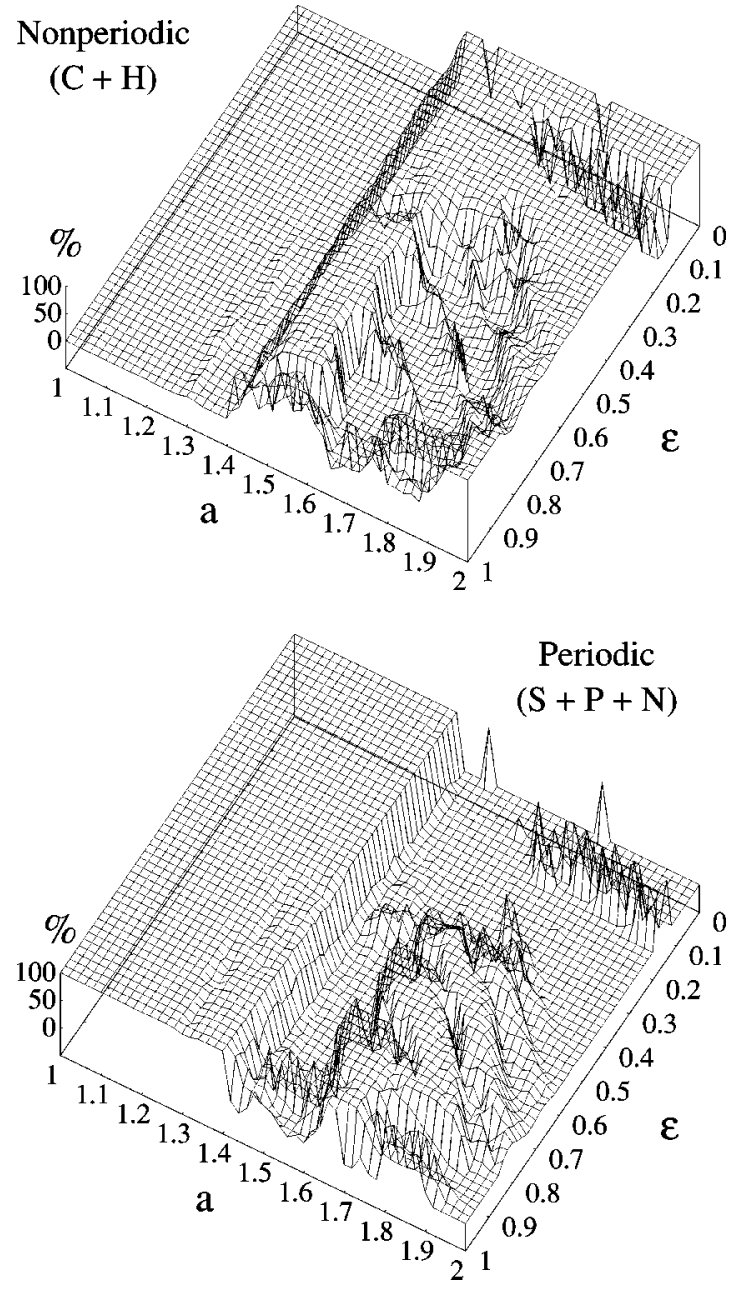

FIG. 5. Distributions of nonperiodic and periodic pattern evolution as a function of nonlinearity $a$ and diffusion $\varepsilon$, in the absence of advection $(\gamma=0)$, for a lattice of $L=64$ sites.

Here, classes $P$ and $N$ have the same distribution, because of the symmetry in the coupling. See Ref. [39] for details on this point.

Figure 6 shows the distributions of periodic and nonperiodic classes in both $(a, L)$ and $(\varepsilon, L)$ spaces. For the $(a, L)$ space we fix $\varepsilon=0.5$, a value for which all classes exist. From the histograms in Fig. 6 one sees that the periodic plateau at $a \lesssim a_{\infty}$ exists for all sizes, but now the upper boundary sightly decreases when $L$ increases, an artifact that could be removed by considering larger transients [41]. We found that for transients about 100 times larger this slight discrepancy disappears.

Nonperiodic PEs predominate for $a_{\infty}<a \leqq 1.6$. For $a$ $\gtrsim 1.6$ one observes mainly periodic PEs, essentially wavelike patterns, moving or not. Interestingly, for this latter region nonperiodic evolutions are found only for specific sizes of the lattice which vary slowly with nonlinearity $a$ and diffusion $\varepsilon$. Moreover, for these specific values we found no periodic evolutions. A possible explanation for this could be the mismatch between the lattice size and the characteristic wavelength $[16,21]$ of the wavelike patterns. Note that for fixed nonlinearity $a$ the sizes $L$ for which nonperiodic PEs are observed seem to be equally spaced. Furthermore, for $L$ $\lesssim 50$, periodic PEs are observed for all values of $a \gtrsim 1.6$, while for $L \gtrsim 50$ they disappear beyond a threshold which decreases with $L$.

Our simulations indicate that moving patterns, either class $P$ or $N$, are not observed for small lattices, of the order of $L \lesssim 10$. This lower limit for observing moving patterns is a general feature of coupled map lattices: there seems to exist a minimum number of sites below which no pattern can move. It would be interesting to find an analytical proof for this observation.

In the $(\varepsilon, L)$ space we fixed $a=1.73$, also a value for which all classes exist. For small coupling strengths, $\varepsilon$ $\lesssim 0.4$, nonperiodic classes predominate, except in two narrow strips localized at $\varepsilon \sim 0.1$ and $\varepsilon \sim 0.3$ where class $S$ is also observed. The predominance of nonperiodic classes at $\varepsilon \lesssim 0.4$ is due to the fact that we chose a value of $a$ for which the local dynamics is chaotic. Choosing a nonlinearity corresponding to periodic local dynamics one would observe mainly class $S$ periodic evolutions.

For $\varepsilon \gtrsim 0.4$ periodic classes predominate although classes $P$ and $N$ may be also observed, except for certain specific lattice sizes, as shown in Fig. 5.

Finally, for larger sizes $L \gtrsim 100$ we observe that all the features described above remain unchanged up to $L \sim 300$, when traveling waves apparently disappear. Transient times required to reach definitive conclusions are quite long for these sizes and we have not attempted to investigate such large lattices.

\section{B. Effects of advection}

We now consider the effect of advection, the contribution which is of great interest for practical applications $[21,24,30]$.

As already demonstrated [21], for coupled map lattices, the range of admissible values of the advection strength depends on the diffusion and is given by [21] $-\varepsilon \leqslant \gamma \leqslant \varepsilon$.

Figures 7 and 8 show histograms of the distribution of PEs in the $(a, \gamma)$ and $(\varepsilon, \gamma)$ spaces, respectively, for $L=64$.

In Fig. 7 we plot the distributions of class $S$ [Fig. 7(a)], class $P$ [Fig. 7(b)], both for $\varepsilon=0.5$, and of nonperiodic classes $C+H$ [Fig. 7(c)-7(e)], for three coupling strengths, $\varepsilon=0.3,0.5$, and 0.7 , respectively. The distribution of class $N$ is symmetric to that of class $P$, with respect to the axis $\gamma$ $=0$.

From Fig. 7 one observes that the periodic plateau at $a$ $\lesssim 1.4$ is composed by static evolutions (class $S$ ) only for weak advection $\gamma \simeq 0$. The periodic plateau also exists when the advection is further increased although only moving patterns are observed. In other words, advection induces static PEs to move. This is true not only for the periodic plateau but also above the accumulation point, i.e., for $a \gtrsim 1.4$.

The predominance of moving patterns is also very pronounced for $1.5 \$ a \lesssim 1.8$. Comparing this region with that observed in the purely diffusive regime (see Fig. 5) one sees that for specific ranges, namely $1.6 \leqq a \leqq 1.8$, PEs which are nonperiodic in the absence of advection become periodic and start to move when advection is present in the system.

Another interesting fact illustrated by Figs. 7(c) and 7(e) concerns nonperiodic PEs in the region delimited by 1.6 

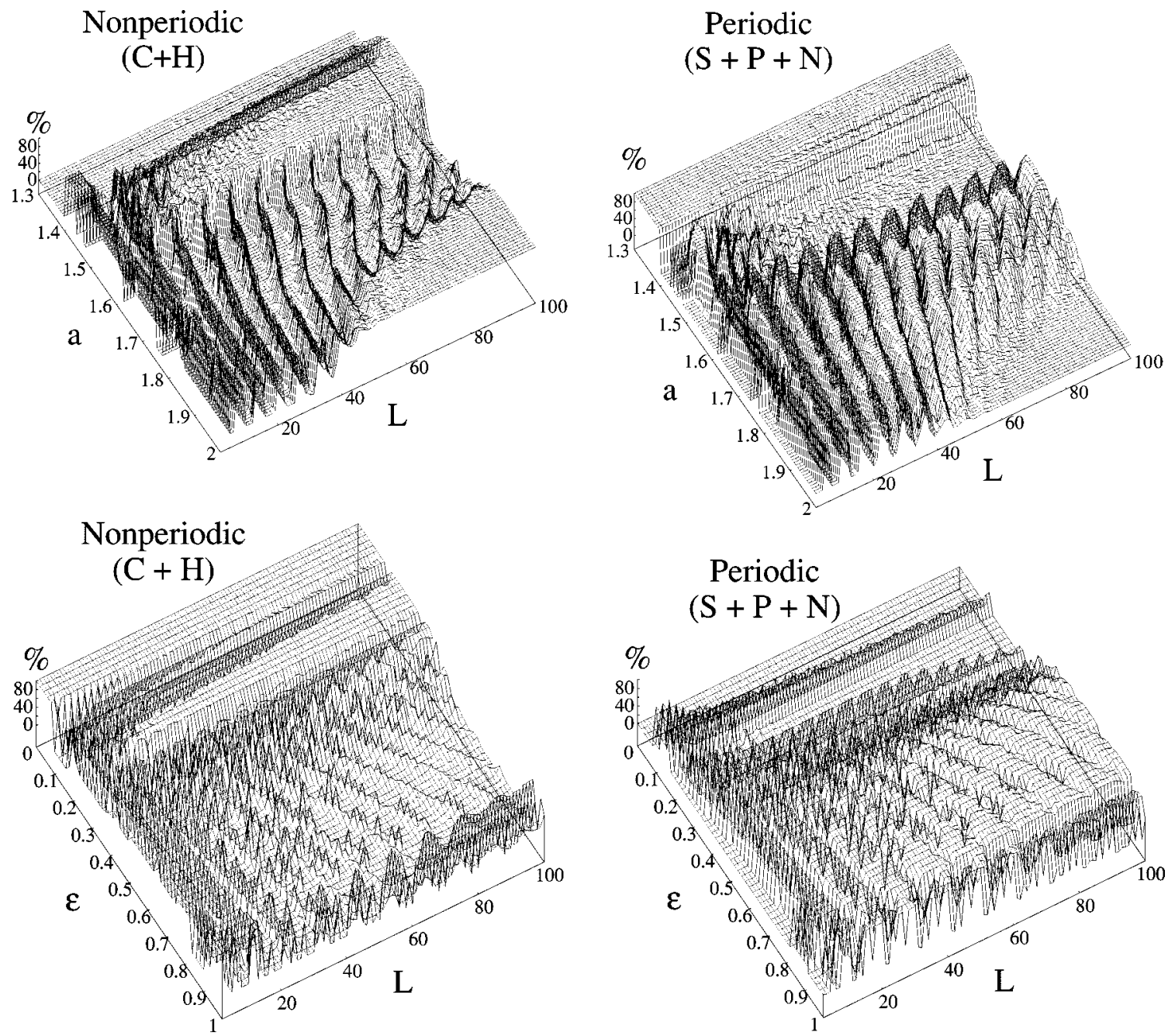

FIG. 6. Distributions of nonperiodic and periodic pattern evolutions as a function of lattice size $L$ for a purely diffusive lattice $(\gamma=0)$. Top: typical distributions as a function of $a$ obtained for $\varepsilon=0.5$. Bottom: typical distributions as a function of $\varepsilon$ obtained for $a=1.73$. These constants are chosen to guarantee the existence of all classes (see text).

$\lesssim a \lesssim 1.85$ for which one observes a pronounced variation if the coupling strength is increased. First, when the coupling strength increases from $\varepsilon=0.3$ to 0.5 nonperiodic PEs turn into periodic ones and move in the lattice. But, if the coupling strength is further increased, strong advection $(\gamma$ $\gtrsim 0.5 \varepsilon$ ) induces those moving patterns to evolve chaotically again. Here, one observes a parabolic shaped plateau beyond which only nonperiodic PEs survive.

The precise mechanism responsible for the switching between nonperiodic and periodic evolutions when advection is present is not yet fully established. However, it is well known $[16,38]$ that the periodicity depends on the quotient $L / \lambda, \lambda$ being the wavelength of the pattern and, as recently reported $[21,30]$, advection induces changes in the characteristic wavelengths. This change of the wavelength may be responsible for the switching between chaotic and periodic behavior. Preliminary studies indicate that the convective Lyapunov exponents seem to be a good tool to discriminate regions in parameter space where advection induces periodicity from regions where it induces chaos.

Figure 8 shows the distribution of classes in $(\varepsilon, \gamma)$ space for class $P$ and nonperiodic $C+H$ classes when the local dynamics $f(x)$ is chaotic, namely for $a=1.7$. The distribution of class $N$ is again symmetric to that of class $P$, while class $S$ is only observed in a narrow strip around $\gamma=0$. The triangular shape of these histograms reflects the condition $-\varepsilon$ $\leqslant \gamma \leqslant \varepsilon$.

As one sees from Fig. 8, nonperiodic evolutions are observed for strong advection $\gamma \sim \varepsilon$, either in the weak or in the strong diffusion regimes. Nonperiodic evolutions almost disappear for other advection strengths, except for specific parabolic curves where a few can be observed.

The central portion of the $\varepsilon$ axis is dominated by moving patterns with velocities approximately given by the advection strength $v=\gamma$, if small random fluctuations are neglected. This is true for chaotic local dynamics, namely, above the accumulation point $a_{\infty}$ of the quadratic map.

Only periodic PEs are observed for periodic local dynamics, i.e., below the accumulation point of the quadratic map. For $\varepsilon \leqq 0.25$ one finds specific intervals, say $-\gamma_{c} \leqslant \gamma \leqslant \gamma_{c}$, where only class $S$ exists. The value of $\gamma_{c}$ is a function of diffusion and can be numerically determined [30,31]. Beyond this interval, static PEs start to move with a velocity obeying a power law, namely, 
TABLE II. Summary of the clasification of the five classes of pattern evolutions of the general diffusive-advective model of Eq. (1). Here $\mathcal{L} \sim 50, a_{2 \rightarrow 4} \sim 1.27, a_{\infty} \sim 1.4011$ is the

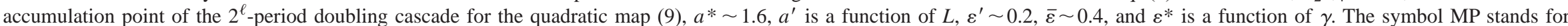
"moving patterns," and denotes class $P$ whenever $\gamma>0$ or class $N$ when $\gamma<0$. Velocities are measured in site/step.

\begin{tabular}{|c|c|c|c|c|c|}
\hline \multirow[t]{2}{*}{$a \backslash \gamma$} & \multirow[b]{2}{*}{$\varepsilon \backslash L$} & \multicolumn{2}{|c|}{ Purely diffusive model $(\gamma=0)$} & \multicolumn{2}{|c|}{ Diffusive-advective model [Eq. (1)] } \\
\hline & & $L<\mathcal{L}$ (Fig.6) & $L>\mathcal{L}$ (Figs. 5 and 6) & $L<\mathcal{L}$ (Fig. 7 and 8 ) & $L>\mathcal{L}$ (Figs. 7-10) \\
\hline \multirow[t]{2}{*}[a^{\prime},2]{} & {$[\bar{\varepsilon}, 1]$} & $\begin{array}{c}S \\
\text { TWs for } L \sim 10 \\
C+H \text { for some } L\end{array}$ & $C+H$ & $\begin{array}{c}C+H \\
\text { TWs with } v \sim \gamma \\
\text { (random fluctuation) }\end{array}$ & $\begin{array}{c}C+H \\
\text { for large }|\gamma|\end{array}$ \\
\hline & {$[0, \bar{\varepsilon}]$} & $\begin{array}{l}C+H \\
\text { Few } S\end{array}$ & $C+H$ & $C+H$ & $\begin{array}{c}C+H \\
\text { for large }|\gamma|\end{array}$ \\
\hline \multirow[t]{2}{*}[a^{*},a^{\prime}]{} & {$[\bar{\varepsilon}, 1]$} & $\begin{array}{c}S \\
\text { TWs for } L \gtrsim 10 \\
C+H \text { for some } L\end{array}$ & $\begin{array}{c}\text { TWs } \\
\left(v \sim 10^{-3}-10^{-2}\right) \\
C+H \text { for some } L\end{array}$ & $\begin{array}{l}\text { TWs with } v \sim \gamma \\
S \text { for small }|\gamma| \\
C+H \text { for some } L\end{array}$ & $\begin{array}{l}\text { TWs with } v \sim \gamma \\
S \text { for small }|\gamma| \\
C+H \text { for some } L\end{array}$ \\
\hline & {$[0, \bar{\varepsilon}]$} & $\begin{array}{c}C+H \\
S \text { for } L \gtrsim 10\end{array}$ & $\begin{array}{c}C+H \\
S \text { for } L \gtrsim 10\end{array}$ & $\begin{array}{l}\text { TWs with } v \sim \gamma \\
S \text { for small }|\gamma|\end{array}$ & $\begin{array}{l}\text { TWs with } v \sim \gamma \\
S \text { for small }|\gamma|\end{array}$ \\
\hline \multirow[t]{2}{*}[a_{\infty},a^{*}]{} & {$[\bar{\varepsilon}, 1]$} & $\begin{array}{c}C+H \\
\text { Few } S \text { for } L \gtrsim 10\end{array}$ & $\begin{array}{c}C+H \\
\text { Few } S \text { for } L \gtrsim 10\end{array}$ & $\begin{array}{c}M P(v \sim 0.1-1) \\
S+C+H \text { for small }|\gamma|\end{array}$ & $\begin{array}{c}M P(v \sim 0.1-1) \\
S+C+H \text { for small }|\gamma|\end{array}$ \\
\hline & {$[0, \bar{\varepsilon}]$} & $C+H$ & $C+H$ & $\begin{array}{c}M P(v \sim 0.1-1) \\
S+C+H \text { for small }|\gamma|\end{array}$ & $\begin{array}{c}M P(v \sim 0.1-1) \\
S+C+H \text { for small }|\gamma|\end{array}$ \\
\hline \multirow[t]{2}{*}[a_{2\rightarrow4},a_{\infty}]{} & {$\left[\varepsilon^{*}, 1\right]$} & $\begin{array}{l}\text { Periodic plateau } \\
P+N\left(v \sim 10^{-4}\right)\end{array}$ & $\begin{array}{l}\text { Periodic plateau } \\
P+N\left(v \sim 10^{-4}\right)\end{array}$ & $\begin{array}{c}M P(v \sim \gamma) \\
S \text { for } L \lesssim 10\end{array}$ & $\begin{array}{c}M P(v \sim \gamma) \\
C+H \text { at } \varepsilon \sim 1\end{array}$ \\
\hline & {$\left[0, \varepsilon^{*}\right]$} & $\begin{array}{l}\text { Periodic plateau } \\
S \text { only }\end{array}$ & $\begin{array}{l}\text { Periodic plateau } \\
\quad S \text { only }\end{array}$ & $\begin{array}{c}M P\left[v \propto \gamma^{\alpha(\varepsilon)}, \text { Eq. (10)] }\right. \\
\quad S \text { (velocity locking) }\end{array}$ & $\begin{array}{c}M P\left[v \propto \gamma^{\alpha(\varepsilon)}, \text { Eq. (10)] }\right. \\
S \text { (velocity locking) } \\
C+H \text { at } \varepsilon \sim 0\end{array}$ \\
\hline \multirow[t]{2}{*}[-0.25,a_{2\rightarrow4}]{} & {$\left[\varepsilon^{\prime}, 1\right]$} & $\begin{array}{l}\text { Periodic plateau } \\
S \text { only }\end{array}$ & $\begin{array}{l}\text { Periodic plateau } \\
\quad S \text { only }\end{array}$ & $\begin{array}{c}M P(v \sim \gamma) \\
S \text { for } L \lesssim 10\end{array}$ & $M P(v \sim \gamma)$ \\
\hline & {$\left[0, \varepsilon^{\prime}\right]$} & $\begin{array}{l}\text { Periodic plateau } \\
S \text { only }\end{array}$ & $\begin{array}{l}\text { Periodic plateau } \\
\quad S \text { only }\end{array}$ & $\begin{array}{c}S \text { (velocity locking) } \\
M P\left[v \propto \gamma^{\alpha(\varepsilon)} \text {, Eq. (10)] }\right. \\
\text { at } a \lesssim 1.1\end{array}$ & $\begin{array}{c}S \text { (velocity locking) } \\
M P\left[v \propto \gamma^{\alpha(\varepsilon)}, \text { Eq. (10) }\right] \\
\text { at } a \leq 1.1\end{array}$ \\
\hline
\end{tabular}



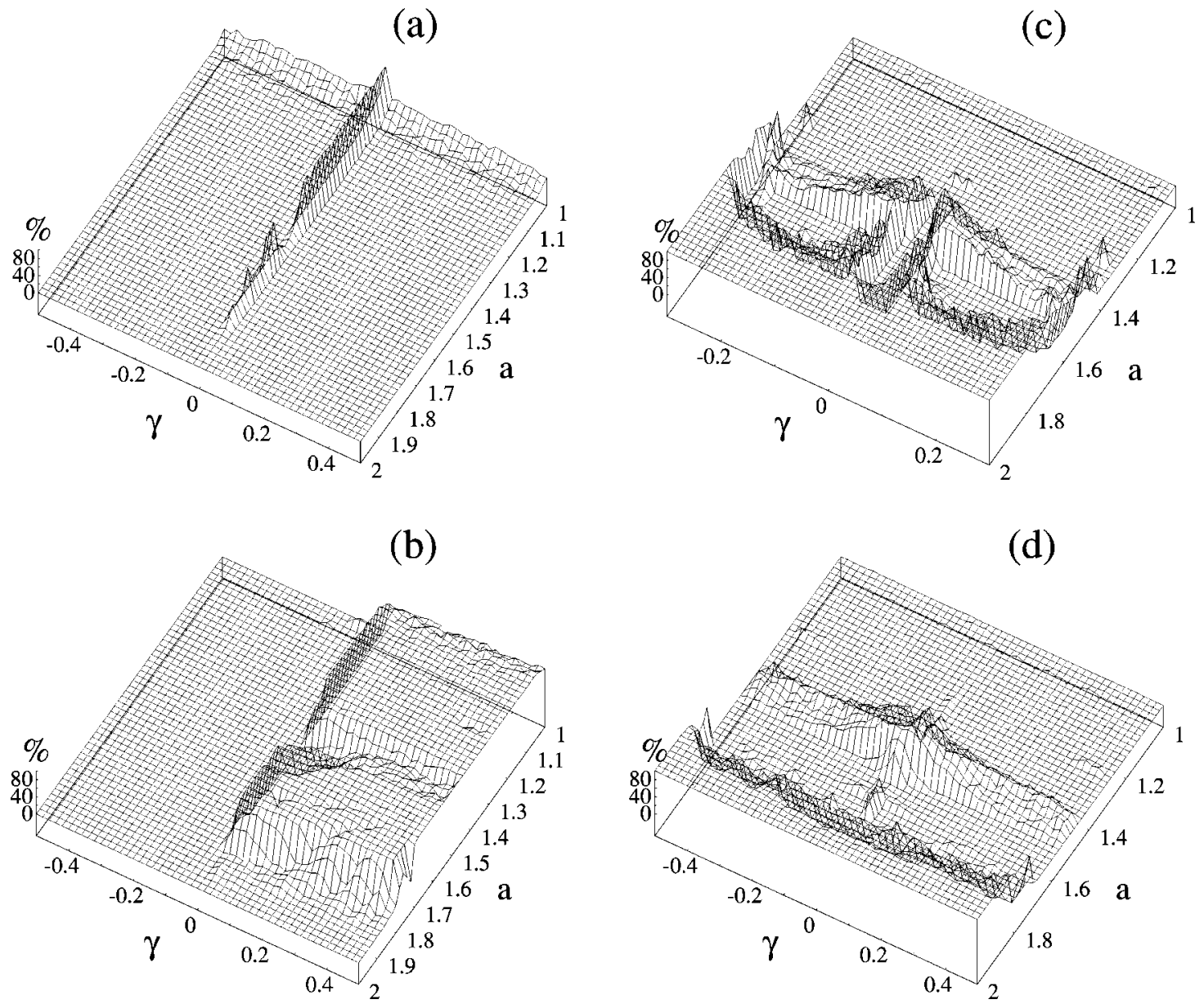

(e)

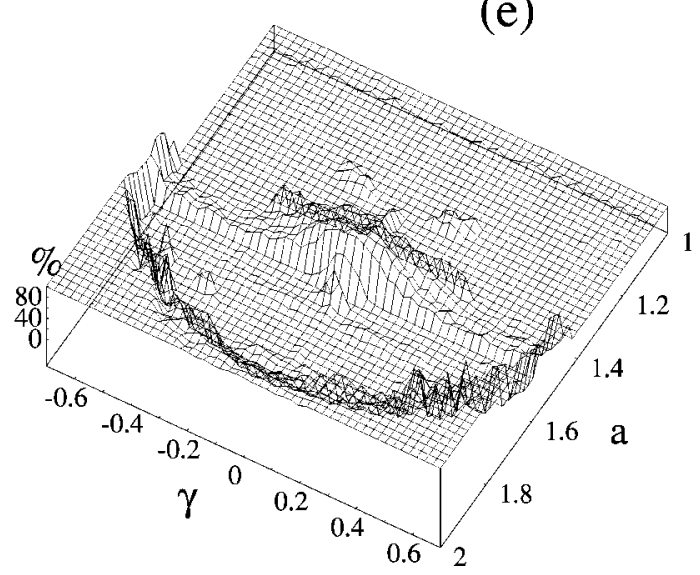

FIG. 7. Distributions of classes as a function of $a$ and $\gamma$. (a) Distribution of class $S$, (b) of class $P$, both for $\varepsilon=0.5$, and of nonperiodic class $C+H$ for (c) $\varepsilon=0.3$, (d) $\varepsilon=0.5$, (e) $\varepsilon=0.7$. In all cases $L=64$.

$$
v=\frac{|\gamma|}{\gamma}\left(\gamma-\gamma_{c}\right)^{\alpha}+\theta
$$

where $\alpha$ is proportional to the diffusion and $\theta$ is a suitable function intended to represent the small-scale fluctuations of the velocity. For $\varepsilon \sim 0.06$ we find $\alpha=0.5$ showing that in this case the velocity of the traveling waves displays the same functional dependence on the advection $\gamma$ as that found be- tween the velocity of gradient flows and the geostrophic velocity [21], a remarkable fact.

When $L$ varies, the dependence on $a$ is similar to that of the purely diffusive regime, with the following differences: (i) patterns which are static in the absence of advection start to move, (ii) for $a \lesssim a_{\infty}$ the number of moving patterns increases with the advection strength. Moreover, for weak diffusion, the region at $a \lesssim 1.1$ where moving patterns are observed, disappears for weak advection $(\gamma \lesssim 0.3 \varepsilon)$, while for 

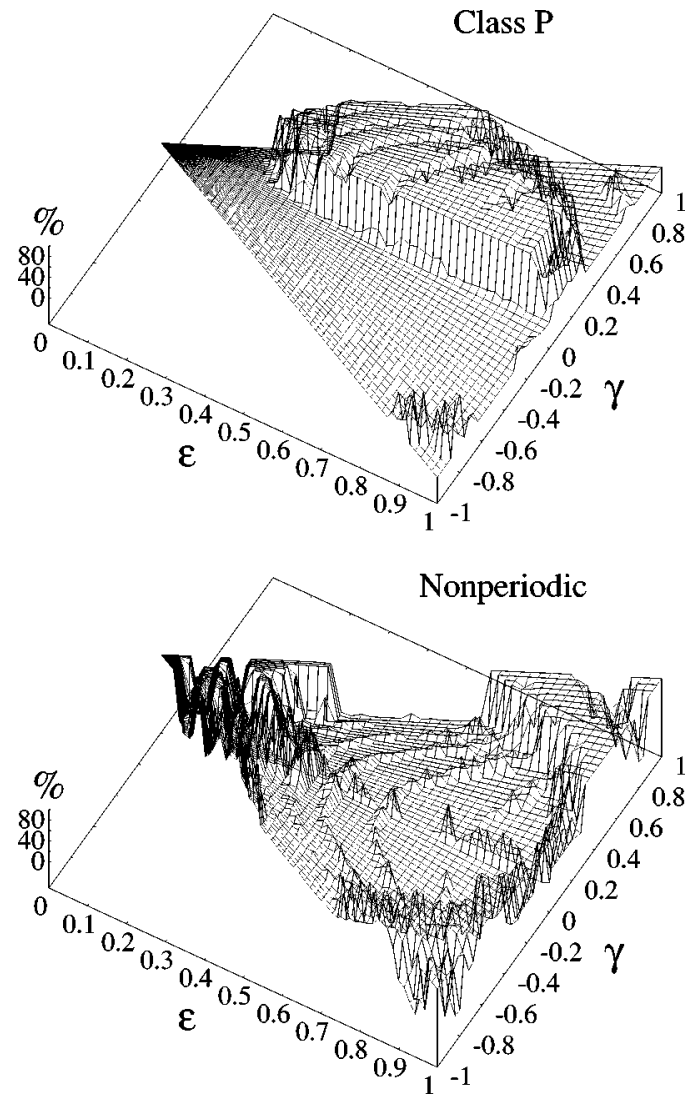

FIG. 8. Distributions of pattern evolutions in the $(\varepsilon, \gamma)$ space $(L=64)$ when local dynamics are chaotic, namely $a=1.7$. Class $N$ displays a distribution symmetric to that of class $P$ with respect to $\gamma=0$. Classes $C$ and $H$ are plotted together as "nonperiodic" distributions.

strong diffusion an increase of advection suppresses moving patterns in the region of high nonlinearity $(a \geqslant 1.8)$.

Figures 9 and 10 illustrate the distribution of patterns as a function of $\varepsilon$ and $\gamma$, respectively, when lattice size $L$ varies.

The distributions in the $(\varepsilon, L)$ space are illustrated in Fig. 9 for class $P$ for $\gamma=0.5 \varepsilon$ and chaotic local dynamics: $a$ $=1.7$ [Fig. 9(a)] and $a=1.9$ [Fig. 9(b)].

From the histogram in Fig. 9(a) a one sees that moving patterns exist for $\varepsilon \gtrsim 0.3$ and also around $\varepsilon \sim 0.1$. Comparing these two regions with those observed in the absence of advection (see Fig. 6) one clearly sees that moving patterns appear for considerably weaker diffusion strengths when advection is present. In particular, for $\varepsilon \gtrsim 0.3$ one finds again only periodic patterns except at particular lattice sizes where nonperiodic evolutions predominate. Apparently, the lattice size displays the same dependence on diffusion as for the purely diffusive regime (compare with Fig. 6).

For very high nonlinearities, e.g., $a=1.9$, the region where moving patterns predominate shrinks with the lattice size and with the nonlinearity $a$, as illustrated in Fig. 9(b). In particular, moving patterns disappear for $L \gtrsim 50$ and for $L \lesssim 10$.

For $a>a_{\infty}$, class $S$ is not observed, while for periodic local dynamics $\left(a<a_{\infty}\right)$, static evolutions predominate for the weak diffusion regime, similarly to what was observed in Fig. 8.
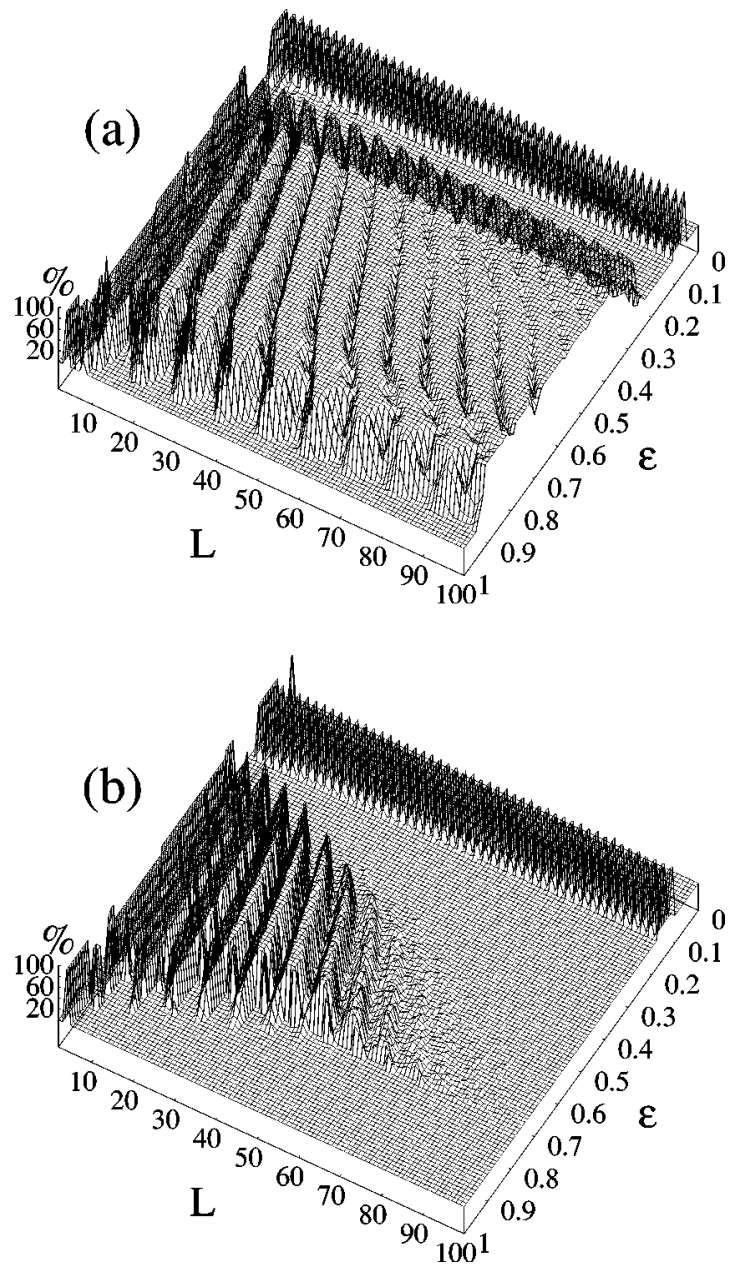

FIG. 9. Distributions of moving patterns of class $P$ as a function of $\varepsilon$ and $L$ for $\gamma=0.5 \varepsilon$ and for chaotic local dynamics, namely (a) $a=1.7$ and (b) $a=1.9$. For values of the advection there are no pattern evolutions belonging to class $N$. For $\gamma=-0.5 \varepsilon$ (not shown) classes $P$ and $N$ interchange distributions.

Finally, the distributions in the $(L, \gamma)$ space are shown in Fig. 10, for $a=1.7$ (chaotic local dynamics) and $\varepsilon=0.7$ (strong diffusion). In the same way when $\varepsilon$ was varied (see Fig. 9), here one observes the predominance of moving patterns, except for very specific lattice sizes, where nonperiodic PEs appear. The specific values of $L$ for each particular case depend of $\gamma$.

Our simulations have shown that for chaotic local dynamics and weak diffusion only nonperiodic PEs are observed, while for periodic local dynamics they are absent and moving patterns (class $P$ and $N$ ) are observed even for strong advection, their density increasing with the diffusion strength.

Table II summarizes the results described in this section, covering the complete domain of parameters $a, \varepsilon, \gamma$ and $L$.

\section{DISCUSSION AND CONCLUSIONS}

This paper described in detail the influence of advection in pattern formation and pattern dynamics in spatial extended systems and classified the possible solutions observed in the 

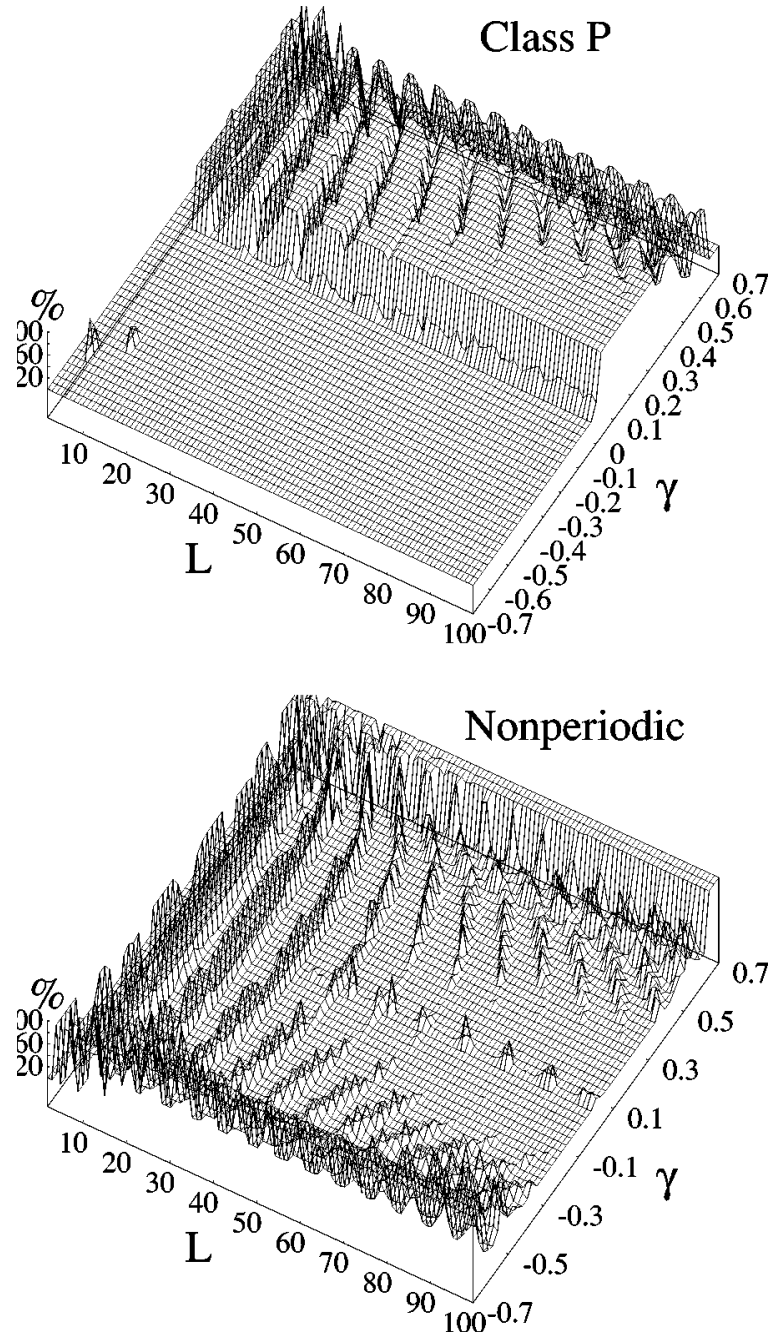

FIG. 10. Distributions of the five classes of pattern evolutions as a function of $\gamma$ and $L$, for chaotic local dynamics $(a=1.7)$ and strong coupling $(\varepsilon=0.7)$. Classes $N$ and $P$ have symmetric distributions with respect to $\gamma=0$.

realistic diffusive-advective lattices of maps. Advection is capable of inducing movement in static patterns. It can also interchange chaotic and periodic behaviors (see Table II). We showed that pattern dynamics in spatially discrete models requires a slight extension of the concept of identical pat- terns, involving interpolated continuous functions instead of amplitudes.

The extended definition allows classifying pattern evolutions into five generic classes (see Fig. 4). The five classes incorporate all possible solutions observed for onedimensional advectively coupled maps. For instance, frozen patterns [16] characterized by periodic sequences of patterns which remain static on the lattice belong to class $S$, wavelike patterns [42], moving fronts [40,43] and traveling waves $[16,40,42-44]$ belong to classes $P$ or $N$, and both frozen random patterns and evolutions with turbulent defects belong to class $C$. Furthermore, our results indicate that these solutions are not transient behaviors in a wide range of parameter values, being robust after transients of up to $10^{7}$ time steps. Moreover, the classes exist for several types of boundary conditions [16] and local dynamics $[39,43,45,46]$. They were even observed for a coupled map lattice with nonlinear heterogeneity [47], i.e., where the local nonlinearity varies in space.

The algorithm used to classify pattern evolutions is based on two quantities only, a temporal periodicity and a displacement indicator, and can be directly applied to any spatially discrete model.

An interesting open question not addressed here is the impact of the periodic windows of the local map (9) in the distribution of the classes. In particular, when advection is absent TWs exist only above the period-3 window, i.e., for $a \gtrsim 1.6$, or between the period- 4 bifurcation value and the accumulation point $a_{\infty}$. Additionally, the velocity locking behavior $[30,31,45]$ found in some parameter domain is not yet fully understood and the precise sizes of the lattice for which one finds a mismatch between the wavelength and the size $L$, inducing chaotic pattern evolutions, should be investigated more closely. We hope to report on these questions soon.

\section{ACKNOWLEDGMENTS}

This work was supported by the bilateral Project No. 077/ 2001 sponsored by CAPES (Brazil) and ICCTI (Portugal), by Fundação para a Ciência e a Tecnologia, Portugal. P.G.L. thanks Fundação para a Ciência e a Tecnologia for financial support and for supporting several stays in Brazil during the last few years. J.A.C.G. thanks Conselho Nacional de Desenvolvimento Científico e Tecnológico, Brazil, for financial support.
[1] J. L. Guisado, F. Jiménez-Morales, and J. M. Guerra, Phys. Rev. E 67, 066708 (2003).

[2] R. Neubecker and O. Jakoby, Phys. Rev. E 67, 066221 (2003); T. Carmon, M. Soljačić, and M. Segev, Phys. Rev. Lett. 89, 183902 (2002).

[3] D. Funfschilling, B. Sammuli, and M. Dennin, Phys. Rev. E 67, 016207 (2003); A. G. Rossberg, ibid. 62, 8114 (2000).

[4] K. Pfeil, M. D. Graham, D. J. Klingenberg, and J. F. Morris, Phys. Rev. Lett. 88, 188301 (2002).

[5] R. Friedrichs, Phys. Rev. E 66, 066215 (2002); J. Richardi, D. Ingert, and M. P. Pileni, ibid. 66, 046306 (2002).
[6] S. Rüdiger, D. G. Míguez, A. P. Muñuzuri, F. Sagués, and J. Casademunt, Phys. Rev. Lett. 90, 128301 (2003).

[7] L. Yang and I. R. Epstein, Phys. Rev. Lett. 90, 178303 (2003).

[8] V. K. Vanag and I. R. Epstein, Phys. Rev. E 67, 066219 (2003).

[9] Y. Shiwa, Phys. Rev. E 67, 026306 (2003).

[10] S. L. Pollicott, P. C. Matthews, and S. M. Cox, Phys. Rev. E 67, 016301 (2003).

[11] M. R. Paul, M. C. Cross, and P. F. Fischer, Phys. Rev. E 66, 046210 (2002).

[12] M. C. Cross and P. C. Hohenberg, Rev. Mod. Phys. 65, 851 
(1993).

[13] M. Howard and A. D. Rutenberg, Phys. Rev. Lett. 90, 128102 (2003).

[14] J. Y. Wakano, S. Maenosono, A. Komoto, N. Eiha, and Y. Yamaguchi, Phys. Rev. Lett. 90, 258102 (2003).

[15] J. Müller and W. van Saarloos, Phys. Rev. E 65, 06111 (2002).

[16] K. Kaneko and I. Tsuda, Complex Systems: Chaos and Beyond (Springer, Berlin, 2000); Theory and Applications of Coupled Map Lattices, edited by K. Kaneko (Wiley, Chichester, 1993).

[17] M. G. Cosenza, M. Pineda, and A. Parravano, Phys. Rev. E 67, 066217 (2003).

[18] T. Shibata and K. Kaneko, Physica D 181, 197 (2003).

[19] C. Hauptmann, H. Touchette, and M. C. Mackey, Phys. Rev. E 67, 026217 (2003).

[20] F. H. Willeboordse, Phys. Rev. E 65, 026202 (2002).

[21] P. G. Lind, J. A. M. Corte-Real, and J. A. C. Gallas, Physica D 168-169, 93 (2002)

[22] S. C. Venkataramani and E. Ott, Phys. Rev. E 63, 046202 (2001).

[23] M. G. Cosenza and K. Tucci, Phys. Rev. E 64, 026208 (2001).

[24] P. G. Lind, S. Titz, T. Kuhlbrodt, J. Corte-Real, J. Kurths, J. A. C. Gallas, and U. Feudel, Int. J. Bifurcation Chaos Appl Sci. Eng. 14, 999 (2004).

[25] A. Pikovsky, M. Rosenblum, and J. Kurths, Synchronization-A Universal Concept in Nonlinear Sciences (Cambridge University Press, Cambridge, 2001).

[26] S. Boccaletti, J. Kurths, G. Osipov, D. L. Valladares, and C. S. Zhou, Phys. Rep. 366, 1 (2002).

[27] P. G. Lind, J. A. M. Corte-Real, and J. A. C. Gallas, Phys. Rev. E 69, 026209 (2004).

[28] L. D. Landau and E. M. Lifshitz, Course of Theoretical Physics, vol. VI-Fluid Mechanics, (Pergamon Press, New Delhi, 1959).
[29] G. Siedler, J. Church, and J. Gould, Ocean Circulation and Climate: Observing and Modeling the Global Ocean (Academic Press, New York, 2001).

[30] P. G. Lind, J. A. M. Corte-Real, and J. A. C. Gallas, Phys. Rev. E 66, 016219 (2002).

[31] P. G. Lind and J. A. C. Gallas, Phys. Scr. (unpublished).

[32] I. S. Aranson, D. Golomb, and H. Sompolinsky, Phys. Rev. Lett. 73, 533 (1992).

[33] Y. Jiang and P. Parmananda, Phys. Rev. E 57, R2499 (1998).

[34] F. Cecconi, A. Crisanti, M. Falcioni, and A. Vulpiani, J. Phys. A 28, 4727 (1995).

[35] T. Bohr, M. H. Jensen, G. Paladin, A. Vulpiani, B. Chirikov, P. Cvitanovic, F. Moss, and H. Swinney, Dynamical Systems Approach to Turbulence (Cambridge University Press, Cambridge, 1998).

[36] K. Kaneko, Physica D 68, 299 (1993).

[37] V. Franceschini and C. Vernia, Phys. Rev. E 57, 2757 (1998).

[38] V. Franceschini, C. Giberti, and C. Vernia, Physica D 164, 28 (2002).

[39] P. G. Lind, J. A. M. Corte-Real, and J. A. C. Gallas, Int. J. Bifurcation Chaos Appl. Sci. Eng. 11, 2647 (2001).

[40] L. A. Bunimovich, V. Franceschini, C. Giberti, and C. Vernia, Physica D 103, 412 (1997).

[41] G. Abramson, Europhys. Lett. 52, 615 (2000).

[42] G. He, A. Lambert, and R. Lima, Physica D 103, 404 (1997).

[43] R. Coutinho and B. Fernandez, Physica D 108, 60 (1997).

[44] R. E. Amritkar and P. M. Gade, Phys. Rev. Lett. 70, 3408 (1993).

[45] R. Carretero-González, D. K. Arrowsmith and F. Vivaldi, Phys. Rev. E 61, 1329 (2000).

[46] W.-X. Qin, Int. J. Bifurcation Chaos Appl. Sci. Eng. 10, 2061 (2000).

[47] P. G. Lind, J. A. M. Corte-Real, and J. A. C. Gallas, Physica A 295, 297 (2001). 